\title{
Increased Alpha-Fetoprotein Serum Level is Predictive for Survival and Recurrence of Hepatocellular Carcinoma in Non-Cirrhotic Livers
}

\author{
Caroline D.M. Witjes $^{\mathrm{a}}$ Wojciech G. Polak ${ }^{\mathrm{a}}$ Cornelis Verhoef $^{\mathrm{b}}$ \\ Ferry A.L.M. Eskens ${ }^{c}$ Roy S. Dwarkasing ${ }^{d} \quad$ Joanne Verheij ${ }^{\mathrm{e}}$ Robert A. de Man $^{f}$ \\ Jan N.M. Ijzermans ${ }^{a}$ \\ a Division of Hepatobiliary and Transplantation Surgery, Department of Surgery, ${ }^{b}$ Division of Surgical Oncology, \\ Department of Surgery, Departments of ${ }^{\mathrm{c}}$ Medical Oncology, ${ }^{\mathrm{d}}$ Radiology, ${ }^{\mathrm{e}}$ Pathology and ${ }^{\mathrm{f}}$ Gastroenterology and \\ Hepatology, Erasmus University Medical Centre, Rotterdam, The Netherlands
}

\section{Key Words}

Hepatocellular carcinoma - Liver · Non-cirrhotic liver .

Survival $\cdot$ Non-viral factors $\cdot$ Risk factors

\begin{abstract}
Background: Hepatocellular carcinoma (HCC) may be diagnosed in the absence of cirrhosis. However, little is known about prognostic factors for the survival of HCC patients with a non-cirrhotic liver in the absence of well-established risk factors. Method: Survival rates and risk factors for survival and recurrence were analysed in all patients diagnosed between 2000 and 2010 with HCC in a non-cirrhotic liver and in the absence of well-established risk factors. Results: Ninety-four patients were analysed. Treatment with curative intent consisted of surgical resection in 43 patients $(46 \%)$ and radiofrequency ablation in 4 patients $(4 \%)$. In patients treated with curative intent and alive 30 days after treatment $(n=40), 1$ - and 5 -year overall survival rates were 95 and $51 \%$, respectively. Patients with a high preoperative a-fetoprotein (AFP) serum level, the presence of microvascular invasion in the resected specimen, a complicated postoperative course and a major resection, due to a great-
\end{abstract}

er tumour volume, had a significantly worse outcome and a higher recurrence rate. In multivariate analysis, a high AFP serum level at presentation was significantly associated with recurrence and a worse survival. Conclusion: HCC presenting in a non-cirrhotic liver in the absence of well-established risk factors has a poor prognosis. Increased AFP serum levels are significantly associated with clinical outcome.

Copyright $\odot 2013$ S. Karger AG, Basel

\section{Introduction}

Hepatocellular carcinoma (HCC) is the fifth most common cancer in the world and the third most common cause of cancer-related mortality [1]. The incidence of HCC is high in Asia and parts of Africa, whereas it is low but increasing in the Western world, even in the Netherlands [1-5].

HCC is the most common primary tumour of the liver and typically develops in the presence of underlying cirrhosis, particularly in high-endemic areas [1]. In low-endemic areas, no cirrhosis is diagnosed in $5-40 \%$ of HCC patients [6-10]. Although many investigators have re-

\section{KARGER}

E-Mail karger@karger.com

www.karger.com/dsu
(C) 2013 S. Karger AG, Basel

$0253-4886 / 13 / 0296-0522 \$ 38.00 / 0$ 
ported on prognostic factors for HCC in a non-cirrhotic liver, they still included patients positive for serological markers of hepatitis $\mathrm{B}$ or $\mathrm{C}$ virus infection, alcohol abuse or haemochromatosis. These are known well-established risk factors for the development of cirrhosis and eventually HCC [11].

In HCC patients with no underlying cirrhosis, the 'normal' quality of the non-tumoural liver parenchyma might make this type of HCC a different entity, with a different aetiology, clinical presentation, management and prognosis [12].

The aim of this retrospective study was to assess the clinical presentation, surgical outcome and prognostic factors in our series of patients who had HCC without the presence of underlying cirrhosis or well-established risk factors for cirrhosis and HCC.

\section{Methods}

\section{Clinical Characteristics}

All patients with HCC diagnosed in a tertiary reference centre between January 2000 and December 2010 were analyzed. Patients included ( $\mathrm{n}=94$ ) had no underlying cirrhosis and none of the wellestablished risk factors for cirrhosis or HCC as determined by negative hepatitis $B$ and $C$ serological markers (anti-hepatitis $C$ virus negative, hepatitis B surface antigen negative, anti-hepatitis B core negative), a negative history of alcohol abuse (more than 6 glasses of alcohol once a week [13]) and absence of haemochromatosis or a metabolic liver disease. Preoperatively, HCC was diagnosed by means of imaging criteria using 2 imaging techniques. If HCC was diagnosed, treatment was discussed in a multidisciplinary meeting according to the American Association for the Study of Liver Diseases guidelines [1]. In all patients, $\alpha$-fetoprotein (AFP) serum levels were assessed. The AFP cut-off point for our hospital is $9 \mathrm{ng} /$ $\mathrm{ml}$. Data on age, gender, size and number of lesions, and the presence and extent of extrahepatic disease were collected.

Patients were primarily assessed for surgical resection; those not amenable for this option were considered for radiofrequency ablation (RFA). Treatment with curative intent was defined as a complete surgical resection, including wedge resection, segment resection, hemihepatectomy and RFA. The presence of a conventional HCC in a non-cirrhotic liver was histologically confirmed postoperatively in all patients. Patients with a fibrolamellar HCC were excluded. Tumour differentiation grade was determined according to the Lauwers classification [14].

Postoperative complications were reported up to 30 days following surgery and were grouped as complicated (pharmacological treatment or intervention needed) or uncomplicated. Patients not amenable for curative therapy were offered palliative options, consisting of either transarterial chemo-embolization, radiotherapy, palliative systemic therapy or best supportive care.

\section{Follow-Up}

After surgical resection, patients were followed up using contrast-enhanced computed tomography or magnetic resonance imaging combined with determination of serum AFP at
3- and 6-month intervals for up to 2 years following treatment. After 2 years, follow-up was continued annually for up to 5 years. HCC recurrence was defined as the appearance of a new focal liver lesion with HCC characteristics, enlargement of lymph nodes in the liver hilum or suspected extrahepatic lesions. The diagnosis was histologically confirmed if necessary. Data regarding survival of patients who were lost to follow-up was obtained from the general practitioner or the civil registration systems.

\section{Statistical Analysis}

Variables were compared using the Mann-Whitney $U$ test, $t$ test or $\chi^{2}$ test, as appropriate. Statistical significance was considered at a $\mathrm{p}$ value $<0.05$. Receiver operating characteristic (ROC) plots were used to define the optimum cut-off point for serum AFP. Multivariate Cox regression analysis was performed to assess the independent prognostic factors. According to the literature, the patient characteristic 'recurrence of disease' has a high negative impact on survival, therefore the analysis was performed within a model adjusting for recurrence. Within this model, preoperative biochemical and postoperative tumour characteristics with a $p$ value $<0.05$ in univariate analysis together with the baseline characteristics age and gender were included to determine the independent contribution of each variable. Survival curves were drawn using the Kaplan-Meier method. Differences between Kaplan-Meier curves were tested using the log-rank test. All analyses were performed using the Statistical Package for Social Sciences 17.0 (SPSS, Chicago, Ill., USA).

\section{Results}

\section{Patient Characteristics}

Ninety-four patients with HCC without underlying cirrhosis and with no established risk factors for HCC were identified. Of these, 46 patients (49\%) were female and the median age was 62 years (range 10-87); median BMI was 23 (range 18-44), and in 12 patients (13\%), diabetes mellitus was present. Table 1 summarizes the patients' biochemical and radiological characteristics.

\section{Outcome}

Forty-three patients (46\%) underwent a surgical resection and 4 (4\%) underwent RFA. Out of 43 patients who underwent a surgical resection, 3 patients died within 30 days after surgery; 2 patients died due to a myocardial infarct and 1 patient died because of an eventful postoperative course secondary to bleeding. Median time to death was 44 months (range $<1-128$ ).

After complete surgical resection $(n=40)$ of a histologically confirmed conventional HCC in a non-cirrhotic liver, the 1-, 3- and 5-year survival rates were 95, 60 and $51 \%$, respectively. Of the 17 patients with an increased serum AFP ( $>9 \mathrm{ng} / \mathrm{ml}), 13$ (76\%) had a normalized serum 
Table 1. Biochemical and radiological characteristics of HCC patients without well-established risk factors

\begin{tabular}{lcc}
\hline Characteristic & $\begin{array}{l}\text { Reference value } \\
\text { of our hospital }\end{array}$ & HCC patients \\
\hline Albumin, g/l & $35-50$ & $41(27-52)$ \\
ASAT, U/l & $0-34$ & $50(16-897)$ \\
ALAT, U/l & $0-44$ & $40(6-461)$ \\
AP, U/l & $0-114$ & $159(55-2,024)$ \\
Bilirubin, $\mu$ mol/l & $0-16$ & $10(4-199)$ \\
AFP, $\mu$ g/l & $0-9$ & $12(1-834,350)$ \\
Number of tumours & & $1(1-7)$ \\
Radiological tumour size, mm & & $98(5-250)$ \\
\hline TNM & & \\
$\quad$ Stage I & & $28(30)$ \\
$\quad$ Stage II & & $16(17)$ \\
$\quad$ Stage IIIA & & $6(13)$ \\
$\quad$ Stage IIIB & & $32(34)$ \\
\hline
\end{tabular}

Data are expressed as medians (range) or numbers of patients (\%). ASAT = Aspartate aminotransferase; ALAT = alanine aminotransferase; $\mathrm{AP}=$ alkaline phosphatase.

AFP after surgical resection. The 4 patients without a normalized serum AFP had HCC metastasis within 5 months after surgery. Fifteen out of 40 patients (38\%) treated by surgical resection had recurrence of HCC during followup. Median time to recurrence was 8 months (range $1-20)$. Of the patients treated with a minor resection $(\mathrm{n}=$ 12), 3 patients had recurrence of HCC. In the case of wedge resections, HCC recurrence was found in the ipsilateral hemi-liver.

Table 2 presents the operative characteristics and postoperative histological characteristics of the patients treated with surgical resection; no characteristics suspicious for hepatocellular adenoma were found.

In 47 patients $(50 \%)$ not amenable for curative treatment, palliative treatment was considered. They received either transarterial chemo-embolization/transarterial embolization (3 patients; 3\%), radiotherapy or systemic chemotherapy (14 patients; $15 \%$ ) or best supportive care (30 patients; 32\%).

\section{Factors Associated with Survival and Recurrence of HCC after Surgical Resection}

The optimal AFP cut-off point selected by ROC plot in 40 patients treated with a surgical resection was between 8.0 and $9.5 \mathrm{ng} / \mathrm{ml}$. Patients with a poor survival after surgical resection more often underwent a major resection, had the presence of microvascular invasion, a moderately
Table 2. Surgical and pathological parameters in HCC patients undergoing surgical resection $(n=40)$

\begin{tabular}{|c|c|}
\hline \multicolumn{2}{|l|}{ Parameter } \\
\hline \multicolumn{2}{|l|}{ Type of resection } \\
\hline \multicolumn{2}{|l|}{ Minor resection } \\
\hline Segment & $11(28)$ \\
\hline \multicolumn{2}{|l|}{ Major resection } \\
\hline Left hemihepatectomy & $10(25)$ \\
\hline Right hemihepatectomy & $16(40)$ \\
\hline Extended left hemihepatectomy & $1(2)$ \\
\hline Extended right hemihepatectomy & $2(5)$ \\
\hline \multicolumn{2}{|l|}{ Postoperative mortality and morbidity } \\
\hline Uncomplicated & $25(63)$ \\
\hline Complicated & $15(37)$ \\
\hline \multicolumn{2}{|l|}{ Fibrosis } \\
\hline F0 & $19(48)$ \\
\hline $\mathrm{F} 1$ & $17(42)$ \\
\hline $\mathrm{F} 2$ & $4(10)$ \\
\hline \multicolumn{2}{|l|}{ Steatosis } \\
\hline G0 & $22(55)$ \\
\hline G1 & $15(38)$ \\
\hline $\mathrm{G} 2$ & $2(5)$ \\
\hline G3 & $1(2)$ \\
\hline \multicolumn{2}{|l|}{ Microvascular invasion } \\
\hline Presence & $22(55)$ \\
\hline Absence & $17(43)$ \\
\hline Undefined & $1(2)$ \\
\hline \multicolumn{2}{|l|}{ Differentiation grade } \\
\hline Good & $8(20)$ \\
\hline Moderate & $22(55)$ \\
\hline Poor & $8(20)$ \\
\hline Undefined & $2(5)$ \\
\hline
\end{tabular}

Data are expressed as numbers of patients, with percentages in parentheses.

or poorly differentiated tumour, a complicated postoperative course, an increased preoperative serum AFP and recurrence of disease (table 3 ). After adjusting for recurrence, in the Cox regression analysis an increased preoperative AFP serum level was shown to be significantly associated with a worse survival (table $4 a$ ).

Patients with recurrence of HCC were characterized by higher preoperative AFP serum levels $(\mathrm{p}=0.003)$ and the presence of microvascular invasion $(\mathrm{p}=0.005)$.

In the Cox regression analysis, an increased preoperative AFP serum level and the presence of microvascular invasion were shown to be significantly associated with HCC recurrence (table 4b).

The 1- and 3-year survival rates of patients with a high and low AFP serum level were 53 and $86 \%$, and 21 and $75 \%$, respectively $(\mathrm{p}=0.010$; fig. 1$)$. 
Table 3. Biochemical, radiological, surgical and pathological characteristics associated with overall survival following surgical resection $(\mathrm{n}=40$; univariate analysis)

\begin{tabular}{|c|c|c|c|}
\hline Characteristic & Survivors & $\begin{array}{l}\text { Non- } \\
\text { survivors }\end{array}$ & $\begin{array}{l}\mathrm{p} \\
\text { value }\end{array}$ \\
\hline AFP & & & 0.010 \\
\hline$\leq 9 \mathrm{ng} / \mathrm{ml}$ & 74 & 26 & \\
\hline$>9 \mathrm{ng} / \mathrm{ml}$ & 47 & 53 & \\
\hline TNM & & & 0.345 \\
\hline Stage I/II & 62 & 38 & \\
\hline Stage III & 62 & 38 & \\
\hline Number of tumours & & & 0.134 \\
\hline 1 & 59 & 41 & \\
\hline$>1$ & 83 & 17 & \\
\hline Radiological tumour size & & & 0.514 \\
\hline$\leq 5 \mathrm{~mm}$ & 80 & 20 & \\
\hline $5-10 \mathrm{~mm}$ & 56 & 44 & \\
\hline$>10 \mathrm{~mm}$ & 57 & 43 & \\
\hline Postoperative course & & & 0.008 \\
\hline Uncomplicated & 72 & 28 & \\
\hline Complicated & 50 & 50 & \\
\hline Type of resection & & & 0.030 \\
\hline Minor & 82 & 18 & \\
\hline Major & 55 & 45 & \\
\hline Fibrosis & & & 0.512 \\
\hline F0 & 68 & 32 & \\
\hline $\mathrm{F} 1$ & 53 & 47 & \\
\hline $\mathrm{F} 2$ & 75 & 25 & \\
\hline Steatosis & & & 0.176 \\
\hline G0 & 55 & 45 & \\
\hline G1 & 63 & 27 & \\
\hline G2 & 50 & 50 & \\
\hline G3 & 100 & 0 & \\
\hline \multicolumn{4}{|l|}{ Microvascular invasion } \\
\hline Presence & 55 & 45 & \\
\hline Absence & 76 & 24 & \\
\hline Differentiation grade & & & 0.048 \\
\hline (histology) & 87 & 13 & \\
\hline Good & 59 & 41 & \\
\hline Moderate & 40 & 60 & \\
\hline \multicolumn{4}{|l|}{ Poor } \\
\hline Recurrence & & & $<0.001$ \\
\hline Present & 13 & 87 & \\
\hline Absent & 82 & 18 & \\
\hline
\end{tabular}

Data are expressed as percentages.

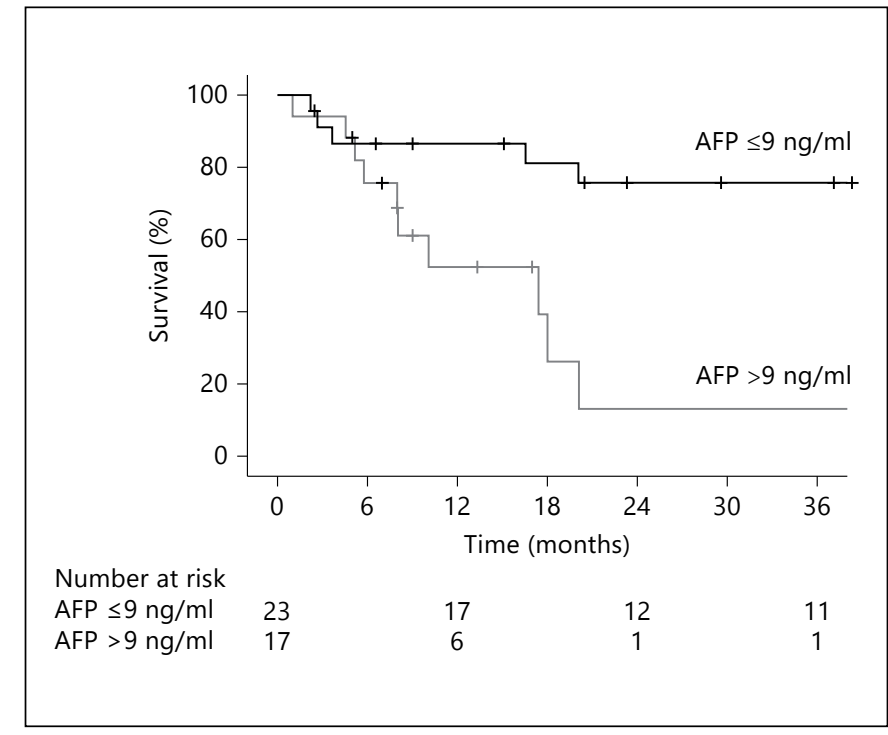

Fig. 1. Overall survival of HCC patients without well-established risk factors starting at 30 days after surgical resection comparing normal versus increased serum levels of AFP.

Table 4. Prognostic factors associated with overall survival and recurrence in multivariate analysis $(n=40)$

a Prognostic factors associated with overall survival

\begin{tabular}{lll}
\hline Characteristic & Hazard ratio & p value \\
\hline Age (years) & $0.98(0.94-1.02)$ & 0.263 \\
Gender & $0.41(0.80-2.07)$ & 0.280 \\
AFP $(\mathrm{ng} / \mathrm{ml})^{1}$ & $1.21(1.02-1.45)$ & 0.034 \\
$\begin{array}{l}\text { Differentiation grade (good vs. } \\
\quad \text { moderate vs. poor) }\end{array}$ & $1.98(0.45-8.72)$ & 0.370 \\
$\begin{array}{l}\text { Microvascular invasion (absent vs. } \\
\quad \text { present) }\end{array}$ & $3.55(0.69-18.33)$ & 0.130 \\
\hline
\end{tabular}

b Prognostic factors associated with recurrence

\begin{tabular}{lll}
\hline Characteristic & Hazard ratio & p value \\
\hline Age (years) & $0.97(0.93-1.01)$ & 0.120 \\
Gender & $1.29(0.39-4.65)$ & 0.702 \\
AFP (ng/ml) & $1.29(1.02-1.63)$ & 0.031 \\
$\begin{array}{l}\text { Differentiation grade (good vs. } \\
\quad \text { moderate vs. poor) }\end{array}$ & $1.05(0.21-5.10)$ & 0.957 \\
$\begin{array}{l}\text { Microvascular invasion (absent vs. } \\
\quad \text { present) }\end{array}$ & $7.03(1.03-47.97)$ & 0.047 \\
\hline \multicolumn{2}{l}{ Values in parentheses represent 95\% confidence limits. } \\
$\quad{ }^{1}$ Continuous variable. \\
\hline
\end{tabular}




\section{Discussion}

In this study, we evaluated the outcome of HCC treatment in patients without well-established risk factors for cirrhosis or HCC. Several studies have explored clinicopathological characteristics in HCC patients without cirrhosis $[1,11,15]$. Although they studied HCC in the absence of cirrhosis, they often included patients with wellestablished risk factors for cirrhosis or HCC or analyzed a small group of patients $[11,16]$. This selection bias will influence overall survival as it is known that, for example, chronic hepatitis B influences survival in HCC patients [17]. We were able to include a large group of patients, all with non-cirrhotic HCC without well-established risk factors, and had the opportunity to study this unique population.

\section{Diagnosis}

In the absence of a screening program developed for patients with liver cirrhosis, early detection in an asymptomatic stage is seldom seen, and diagnosis of non-cirrhotic HCC is usually made at an advanced stage with large tumours [18]. By then, many patients have already developed an unresectable tumour, either because of tumour size, anatomical localization or the presence of multiple bilobar intrahepatic metastases [19]. In the present study, nearly $50 \%$ of the patients referred with HCC without liver cirrhosis and well-established risk factors were beyond curative surgical options. The studied population was homogenous for conventional HCC; none of the patients had a fibrolamellar HCC.

\section{Survival and Recurrence}

In our population of HCC patients without well-established risk factors, the survival rates were comparable with those of patients with Child-Pugh A liver cirrhosis treated with surgical resection (5-year survival rate $50 \%)$, with similar associated factors $[1,20]$. As the optimal AFP cut-off point selected by ROC plot was between 8.0 and $9.5 \mathrm{ng} / \mathrm{ml}$, the AFP cut-off point of our hospital $(9 \mathrm{ng} / \mathrm{ml})$ was an adequate cut-off point. Although AFP as a serological marker for HCC in the screening and surveillance of patients with cirrhosis is debated [21], AFP seems a useful marker in patients with HCC without well-established risk factors, especially when the AFP serum level is increased. It is likely that the 4 patients without normalization of AFP who had metastases within 5 months of surgery already had metastatic disease at surgery that was not identified by imaging due to small size.
According to the literature, little is known about the value of the AFP serum level in patients with HCC without well-established risk factors. Our study displays the presence of an increased AFP serum level preoperatively to be the most important factor associated with a worse outcome and recurrence of HCC in multivariate analysis. If the AFP serum level is increased in patients who have to undergo a high-risk operation, e.g. an extended hemihepatectomy, or in patients with many comorbidities, it might be worth reconsidering the highrisk treatment.

\section{Therapy Considerations}

If a surgical resection is performed with curative intent in a non-cirrhotic liver, $38 \%$ of patients suffer from recurrence of disease. Solitary recurrence might benefit from repeated resection, but in most patients recurrence after primary resection will be multifocal because of intrahepatic dissemination from the primary tumour [1]. Out of 15 patients with recurrence after treatment with curative intent, 3 had recurrence after a local surgical resection. Their disease recurrence appeared as a tumour in the ipsilateral hemi-liver, whereas the contralateral hemi-liver was free of tumour recurrence. Thus, in HCC patients with a well-preserved liver function, there might be a preference to perform a major resection because, after minor resections, patients tend to more often have recurrence in the ipsilateral hemi-liver. In the absence of well-established risk factors, the presence of a small satellite tumour, too small to detect on imaging, might cause tumour recurrence in the ipsilateral liver lobe.

Until now, liver transplantation has been a therapy considered for cirrhotic patients only. In patients with underlying cirrhosis treated with liver transplantation, 5 -year survival rates up to $70 \%$ are reported [1]. This higher survival rate makes transplantation a treatment option worth considering for HCC patients without well-established risk factors [19]. A recent study by Mergental et al. [22] reported the largest series of patients transplanted for non-cirrhotic HCC. After analysing 105 patients, they concluded that liver transplantation may be justified as a curative treatment option in a selected group of patients with unresectable HCC in an otherwise normal liver [22]. The Milan criteria and the algorithms used for HCC in cirrhotic livers are probably not applicable to HCC in a non-cirrhotic liver without well-established risk factors, because it is a different disease with (probably) different aetiology and pathogenesis [23, 24]. Mergental et al. [22] reported tumour size not to be an
Witjes/Polak/Verhoef/Eskens/ Dwarkasing/Verheij/de Man/Ijzermans 
important risk factor for post-transplant survival in patients with a non-cirrhotic HCC. Based on these findings, we propose the following treatment strategy for patients with unresectable HCC in a non-cirrhotic liver without well-established risk factors. Patients with extrahepatic spreading or gross vascular involvement should be strictly excluded from liver transplantation. If a biopsy is performed and the presence of a moderately or poorly differentiated tumour with microvascular invasion is noted, caution is needed, especially when the preoperative serum AFP level is high. A large tumour size per se (outside the Milan criteria) should not be seen as a strict contraindication for considering liver transplantation in patients with HCC without well-established risk factors.

This study evaluating a single institution's experience of HCC patients without well-established risk factors is influenced by confounding factors, as the data were acquired retrospectively and might be influenced by patient selection. However, as patients with HCC without underlying cirrhosis are relatively rare, no previous study was able to describe such a large cohort. Although our study was based on a small number of patients $(n=40)$, still we analyzed the largest cohort presented so far. Although the study population is unique, because some of our conclusions are based on a small number of patients ( 3 patients with local recurrence), experiences from other centres are needed. However, based on our observations, the absence of well-established risk factors seems to have less impact on final outcome parameters in HCC patients. One can speculate whether the presence of cirrhosis is an independent prognostic parameter or whether the overall assessment of HCC and the recurrence of HCC after treatment with curative intent determine the final outcome in patients with HCC. Therefore, expansion of treatment options by liver transplantation in non-cirrhotic patients is worth reconsidering.

In conclusion, this retrospective analysis of a large cohort of HCC patients without well-established risk factors shows that they were diagnosed at an advanced stage of disease. If surgical resection is performed, good results can be achieved. The higher the preoperative AFP serum level, the worse the outcome. Patients might benefit from major resections, as disease recurrence often appears in the ipsilateral liver lobe instead of the contralateral liver lobe. Although cirrhosis is absent, liver transplantation as a treatment option for HCC patients without well-established risk factors is worth considering and should be further explored.

\section{Disclosure Statement}

Nothing to declare.

\section{References}

1 Bruix J, Sherman M; Practice Guidelines Committee, American Association for the Study of Liver Diseases: Management of hepatocellular carcinoma: an update. Hepatology 2011;53:1020-1022.

-2 Taylor-Robinson SD, Foster GR, Arora A, Hargreaves S, Thomas HC: Increase in primary liver cancer in the UK, 1979-94. Lancet 1997;350:1142-1143.

3 Deuffic S, Poynard T, Buffat L, Valleron AJ: Trends in primary liver cancer. Lancet 1998; 351:214-215.

4 El-Serag HB, Mason AC: Rising incidence of hepatocellular carcinoma in the United States. N Engl J Med 1999;340:745-750.

$\checkmark 5$ Witjes CDM, Karim-Kos HE, Visser O, van den Akker SAW, de Vries E, Ijzermans JNM, et al: Hepatocellular carcinoma in a low endemic area: rising incidence and improved survival. Eur J Gastroenterol Hepatol 2012;24:450-457.

6 Verhoef C, de Man RA, Zondervan PE, Eijkemans MJC, Tilanus HW, Ijzermans JNM: Good outcomes after resection of large hepatocellular carcinoma in the non-cirrhotic liver. Dig Surg 2004;21:380-386.
7 Nzeako UC, Goodman ZD, Ishak KG: Hepatocellular carcinoma in cirrhotic and noncirrhotic livers. A clinico-histopathologic study of 804 North American patients. Am J Clin Pathol 1996;105:65-75.

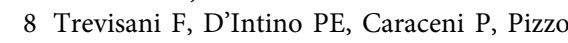
M, Stefanini GF, Mazziotti A, et al: Etiologic factors and clinical presentation of hepatocellular carcinoma. Differences between cirrhotic and noncirrhotic Italian patients. Cancer 1995;75:2220-2232.

-9 Van Roey G, Fevery J, van Steenbergen W: Hepatocellular carcinoma in Belgium: clinical and virological characteristics of 154 consecutive cirrhotic and non-cirrhotic patients. Eur J Gastoenterol Hepatol 2000;12:61-66.

10 Kubicka S, Rudolph KL, Hanke M, Tietze MK, Tillmann HL, Trautwein C, et al: Hepatocellular carcinoma in Germany: a retrospective epidemiological study from a lowendemic area. Liver 2000;20:312-318.
11 Nagasue N, Ono T, Yamanoi A, Kohno H, ElAssal ON, Taniura H, et al: Prognostic factors and survival after hepatic resection for hepatocellular carcinoma without cirrhosis. Br J Surg 2001;88:515-522.

12 Alkofer B, Lepennec V, Chice L: Hepatocellular cancer in the non-cirrhotic liver. J Visc Surg 2001;148:3-11.

13 Statistics Netherlands: Healthcare use and hospital admission statistics in the Netherlands. http://statline.cbs.nl/StatWeb/selectio $\mathrm{n} / ? \mathrm{DM}=\mathrm{SLNL} \& \mathrm{PA}=71860 \mathrm{NED} \& \mathrm{VW}=\mathrm{T}$ (accessed April 24, 2009).

14 Lauwers GY, Terris B, Balis UJ, Batts KP, Regimbeau JM, Chang Y, et al: Prognostic histologic indicators of curatively resected hepatocellular carcinomas: a multi-institutional analysis of 425 patients with definition of a histologic prognostic index. Am J Surg Pathol 2002;26:25-34.

$\checkmark 15$ Smoot RL, Nagorney DM, Chandan VS, Que FG, Schleck CD, Harmsen WS, et al: Resection of hepatocellular carcinoma in patients without cirrhosis. Br J Surg 2011;98:697-703.
Hepatocellular Carcinoma in NonCirrhotic Livers 
16 Lubrano J, Huet E, Tsilividis B, François A, Goria O, Riachi G, et al: Long-term outcome of liver resection for hepatocellular carcinoma in noncirrhotic nonfibrotic liver with no viral hepatitis or alcohol abuse. World J Surg 2008;22:104-109.

17 Hung IF, Poon RT, Lai CL, Fung J, Fan ST, Yuen MF: Recurrence of hepatitis B-related hepatocellular carcinoma is associated with high viral load at time of resection. Am J Gastroenterol 2008;103:1663-1673.

18 Trevisani F, Frigerio M, Santi V, Grignaschi A, Bernardi M: Hepatocellular carcinoma in non-cirrhotic liver: a reappraisal. Dig Liver Dis 2010;42:341-347.
19 Mergental H, Porte J: Liver transplantation for unresectable hepatocellular carcinoma in patients without liver cirrhosis. Transplant Int 2010;23:662-667.

20 Lang H, Sotiropoulos GC, Dömland M, Frühauf NR, Paul A, Hüsting J, et al: Liver resection for hepatocellular carcinoma in non-cirrhotic liver without underlying viral hepatitis. Br J Surg 2005;92:198-202.

21 Giannini EG, Farinati F, Trevisani F: Alphafetoprotein in hepatocellular carcinoma surveillance: wake not the dead. Hepatology 2011;1:367-377.
22 Mergental H, Adam R, Ericzon BG, Kalicinski P, Mühlbacher F, Höckerstedt K, et al: Liver transplantation for unresectable hepatocellular carcinoma in normal livers. J Hepatol 2012;57:297-305.

23 Mazzaferro V, Regalia E, Doci R, Andreola S, Pulvirenti A, Montalto F, et al: Liver transplantation for the treatment of small hepatocellular carcinomas in patients with cirrhosis. N Engl J Med 1996;334:693-699.

24 Wong N, Lai P, Lee SW, Fan S, Pang E, Liew $\mathrm{CT}$, et al: Assessment of genetic changes in hepatocellular carcinoma by comparative genomic hybridization analysis: relationship to disease stage, tumor size, and cirrhosis. Am J Pathol 1999;154:37-43. 